\title{
A Parallel Structure Exploiting Factorization Algorithm with Applications to Model Predictive Control
}

Isak Nielsen and Daniel Axehill

\author{
Linköping University Post Print
}

\section{Tweet}

N.B.: When citing this work, cite the original article.

(C2015 IEEE. Personal use of this material is permitted. However, permission to reprint/republish this material for advertising or promotional purposes or for creating new collective works for resale or redistribution to servers or lists, or to reuse any copyrighted component of this work in other works must be obtained from the IEEE.

Isak Nielsen and Daniel Axehill, A Parallel Structure Exploiting Factorization Algorithm with Applications to Model Predictive Control, 2015, Proceedings of the 54th IEEE Conference on Decision and Control., 3932-3938.

Postprint available at: Linköping University Electronic Press

http://urn.kb.se/resolve?urn=urn:nbn:se:liu:diva-123856 


\title{
A Parallel Structure Exploiting Factorization Algorithm with Applications to Model Predictive Control
}

\author{
Isak Nielsen and Daniel Axehill
}

\begin{abstract}
In Model Predictive Control (MPC) the control signal is computed by solving a constrained finite-time optimal control (CFTOC) problem at each sample in the control loop. The CFTOC problem can be solved by, e.g., interior-point or active-set methods, where the main computational effort in both methods is known to be the computation of the search direction, i.e., the Newton step. This is often done using generic sparsity exploiting algorithms or serial Riccati recursions, but as parallel hardware is becoming more commonly available the need for parallel algorithms for computing the Newton step is increasing. In this paper a tailored, non-iterative parallel algorithm for computing the Newton step using the Riccati recursion is presented. The algorithm exploits the special structure of the Karush-Kuhn-Tucker system for a CFTOC problem. As a result it is possible to obtain logarithmic complexity growth in the prediction horizon length, which can be used to reduce the computation time for popular state-of-the-art MPC algorithms when applied to what is today considered as challenging control problems.
\end{abstract}

\section{INTRODUCTION}

One of the most widely used advanced control strategies in industry today is Model Predictive Control (MPC), and some important reasons for its success include that it can handle multivariable systems and constraints on control signals and state variables in a structured way. Each sample of the MPC control loop requires the solution of a constrained finite-time optimal control (CFTOC) problem on-line, which creates a need for efficient optimization algorithms. Depending on the type of system and problem formulation, the MPC problem can be of different types. The most common variants are linear MPC, nonlinear MPC and hybrid MPC. In most cases, the effort spent when solving the CFTOC problem boils down to solving Newton-system-like equations that correspond to an unconstrained finite-time optimal control (UFTOC) problem. Hence, much focus in research has been spent on solving this type of system of equations efficiently when it has the special form from MPC, see e.g. [1]-[7].

In recent years, the need for parallel algorithms for solving the MPC problem has increased, and much effort in research has been spent on this topic, [8]. In [9] an extended Parallel Cyclic Reduction algorithm is used to reduce the computation of the UFTOC problem to smaller systems of equations that are solved in parallel. The computational complexity of this algorithm is reported to be $\mathcal{O}(\log N)$, where $N$ is the prediction horizon. In [10] and [11] a time-splitting approach to split the prediction horizon into blocks is adopted. The subproblems are connected through common variables and are solved in parallel using Schur complements. The common

I. Nielsen and D. Axehill are with the Division of Automatic Control, Linköping University, SE-58183 Linköping, Sweden, isak.nielsen@liu.se, daniel.axehill@liu.se. variables are computed via a consensus step where a dense system of equations involving all common variables is solved serially. In [12] a splitting method based on Alternating Direction Method of Multipliers (ADMM) is used, where some steps of the algorithm can be computed in parallel. In [13] an iterative three-set splitting quadratic programming (QP) solver is developed. In this method several simpler subproblems are computed in parallel and a consensus step using ADMM is performed to obtain the final solution. In [14], [15] a tailored algorithm for solving the Newton step directly (non-iteratively) in parallel for MPC is presented. In that work several subproblems are solved parametrically in parallel by introducing constraints on the terminal states. However, the structure is not exploited when the subproblems are solved. The results in [14] has been extended in [16] to cover more general problems.

The main contribution in this paper is the introduction of theory and algorithms for computing the Riccati recursion in parallel, which can be used to solve a UFTOC problem in parallel. Furthermore, the performance of the algorithms are illustrated using an ANSI-C implementation of the proposed algorithms that is executed truly in parallel on a physical cluster. The new algorithms are tailored for UFTOC problems that are related to MPC problems and exploit the special structure of the KKT system for such problems. The classical serial Riccati method exploits the causality of the problem and for that reason it is not obvious that it can be split and parallelized in time, especially without involving some form of iterative consensus step. In this paper, it is shown that it in fact can be performed, and how it can be performed. The main idea is to exploit the problem structure in time and divide the UFTOC problem in smaller subproblems along the prediction horizon. Consensus is reached directly (non-iteratively) by solving a master problem. This overall structure is similar to what is done in [14], but the conceptual difference in this paper is how to solve the subproblems to exploit structure and hence improving performance and reducing communication loads. A more detailed presentation of the work in [14] and in this paper is given in [17].

In this paper $\mathbb{S}_{++}^{n}\left(\mathbb{S}_{+}^{n}\right)$ denotes symmetric positive (semi) definite matrices with $n$ columns, $\mathbb{Z}_{i, j} \triangleq\{i, i+1, \ldots, j\}$ and symbols in sans-serif font (e.g. $\mathrm{x})$ denote vectors or matrices of stacked components. Furthemore, $I$ denotes the identity matrix of appropriate dimension, and the product operator is defined as

$$
\prod_{t=t_{1}}^{t_{2}} A_{t} \triangleq\left\{\begin{array}{l}
A_{t_{2}} \cdots A_{t_{1}}, t_{1} \leq t_{2} \\
I, t_{1}>t_{2} .
\end{array}\right.
$$




\section{Problem Formulation}

Some of the most common variants of MPC are linear MPC, nonlinear MPC and hybrid MPC. The corresponding CFTOC problems can be solved using different types of optimization methods, where two commonly used types are interiorpoint (IP) methods and active-set (AS) methods. The main computational effort in both types is spent while solving a sequence of problems consisting of Newton-system-like equations that often corresponds to a UFTOC problem (or to a problem with similar structure) [2], [4], [18]. This UFTOC problem will be denoted $\mathcal{P}(N)$, and has the structure

$$
\begin{aligned}
\min _{\mathrm{x}, \mathrm{u}} . & \sum_{t=0}^{N-1}\left(\frac{1}{2}\left[\begin{array}{l}
x_{t} \\
u_{t}
\end{array}\right]^{T} Q_{t}\left[\begin{array}{l}
x_{t} \\
u_{t}
\end{array}\right]+l_{t}^{T}\left[\begin{array}{l}
x_{t} \\
u_{t}
\end{array}\right]+c_{t}\right) \\
& +\frac{1}{2} x_{N}^{T} Q_{x, N} x_{N}+l_{N}^{T} x_{N}+c_{N} \\
\text { s.t. } & x_{0}=\bar{x}_{0} \\
& x_{t+1}=A_{t} x_{t}+B_{t} u_{t}+a_{t}, t \in \mathbb{Z}_{0, N-1},
\end{aligned}
$$

where $N$ is the prediction horizon length, $x_{t} \in \mathbb{R}^{n_{x}}$ are the states and $u_{t} \in \mathbb{R}^{n_{u}}$ are the control signals. The equality constraints enforce the affine dynamics equations of the system. Let the cost function satisfy Assumption 1 for all $t$

Assumption 1:

$Q_{t} \triangleq\left[\begin{array}{cc}Q_{x, t} & Q_{x u, t} \\ Q_{x u, t}^{T} & Q_{u, t}\end{array}\right] \in \mathbb{S}_{+}^{n_{x}+n_{u}}, Q_{u, t} \in \mathbb{S}_{++}^{n_{u}}, Q_{x, N} \in \mathbb{S}_{+}^{n_{x}}$

Remark 1: In both IP and AS methods the solution to the original CFTOC problem is obtained by solving a sequence of UFTOC problems in the form in (2). The number of problems in this sequence is independent of how these UFTOC problems are solved. Since the main computation time is consumed when the UFTOC problems are solved, the overall relative performance gain for solving the entire sequence of problems in order to solve the CFTOC problem is roughly the same as the relative performance gain obtained when solving a single UFTOC problem.

\section{SERIAL RicCATI RECURSiON}

The optimal solution to the UFTOC problem (2) is computed by solving the set of linear equations given by the associated Karush-Kuhn-Tucker (KKT) system. For this problem structure, the KKT system has a very special form that is almost block diagonal and it is well known that it can be factored efficiently using a Riccati factorization [5]. The Riccati factorization is used to factor the KKT coefficient matrix, followed by backward and forward recursions to compute the primal and dual variables. Using the Riccati recursion to solve the KKT system reduces the computational complexity from roughly $\mathcal{O}\left(N^{2}\right)-\mathcal{O}\left(N^{3}\right)$ to $\mathcal{O}(N)$ compared to solvers that do not exploit sparsity. The Riccati recursion is given by algorithms 1-3, where $F_{t}, P_{t} \in \mathbb{S}_{+}^{n_{x}}, G_{t} \in \mathbb{S}_{++}^{n_{u}}$, $H_{t} \in \mathbb{R}^{n_{x} \times n_{u}}$ and $K_{t} \in \mathbb{R}^{n_{u} \times n_{x}}$, [5]. For more background information on Riccati factorizations, see, e.g., [1], [2] or [5].
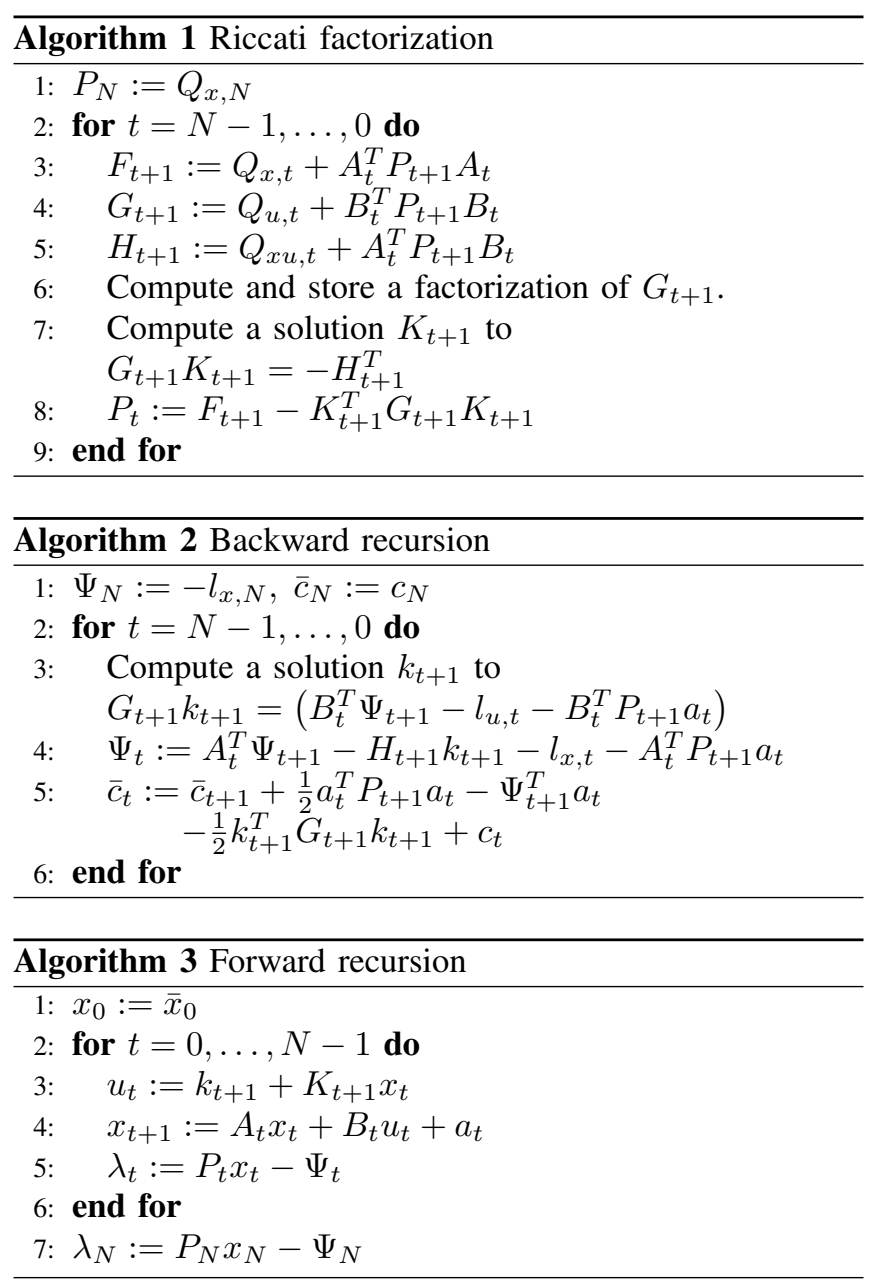

\section{Problem Decomposition and Reduction}

By examining algorithms 1 and 2, it can be seen that given $P_{\bar{t}}, \Psi_{\bar{t}}$ and $\bar{c}_{\bar{t}}$ the factorization and backward recursion can be computed for $0 \leq t \leq \bar{t}$. If these are computed, it follows from Algorithm 3 that given $x_{\bar{s}}$ the forward recursion can be computed for $\bar{s} \leq t \leq N$. Hence, if $P_{\bar{t}}, \Psi_{\bar{t}}, \bar{c}_{\bar{t}}$ and $x_{\bar{s}}$ are known, the Riccati recursion and the primal and dual optimal solution for the interval $\bar{s} \leq t \leq \bar{t}$ can be computed using information from this interval only. As a consequence, provided that $P_{t_{i+1}}, \Psi_{t_{i+1}}, \bar{c}_{t_{i+1}}$ and $x_{t_{i}}$ are known for $i \in \mathbb{Z}_{0, p}$ for some $p$, it is possible to compute the Riccati recursion and the primal and dual solution in each interval $t_{i} \leq t \leq t_{i+1}$ with $i \in \mathbb{Z}_{0, p}$ independently from the other intervals. This property will be exploited in Section IV-A.

\section{A. Splitting into independent parts}

To decompose the UFTOC problem (2) the prediction horizon is divided into $p+1$ intervals, or batches. Now introduce the batch-wise variables $x_{i}$ and $u_{i}$ as

$$
\begin{aligned}
& \mathrm{x}_{\mathrm{i}}=\left[\begin{array}{lll}
x_{0, i}^{T} & \cdots & x_{N_{i}, i}^{T}
\end{array}\right]^{T} \triangleq\left[\begin{array}{lll}
x_{t_{i}}^{T} & \cdots & x_{t_{i+1}}^{T}
\end{array}\right]^{T}, \\
& \mathbf{u}_{\mathrm{i}}=\left[\begin{array}{lll}
u_{0, i}^{T} & \cdots & u_{N_{i}-1, i}^{T}
\end{array}\right]^{T} \triangleq\left[\begin{array}{lll}
u_{t_{i}}^{T} & \cdots & u_{t_{i+1}-1}^{T}
\end{array}\right]^{T},
\end{aligned}
$$

where $N_{i}$ is the length of batch $i, t_{0}=0$ and $x_{N_{i}, i}=x_{0, i+1}$.

By following the reasoning in the introduction of this section it is possible to compute the Riccati recursion and the optimal value in batch $i$ if $\hat{x}_{i} \triangleq x_{t_{i}}, \hat{P}_{i+1} \triangleq P_{t_{i+1}}$, 
$\hat{\Psi}_{i+1} \triangleq \Psi_{t_{i+1}}$ and $\hat{c}_{i+1} \triangleq \bar{c}_{t_{i+1}}$ are known. Hence, if these variables are known for all batches $i \in \mathbb{Z}_{0, p}$, the solution to the original UFTOC problem (2) can be computed from $p+1$ independent subproblems in the UFTOC form

$$
\begin{aligned}
\min _{\mathrm{x}_{\mathrm{i}}, \mathrm{u} i} & \sum_{t=0}^{N_{i}-1}\left(\frac{1}{2}\left[\begin{array}{l}
x_{t, i} \\
u_{t, i}
\end{array}\right]^{T} Q_{t, i}\left[\begin{array}{l}
x_{t, i} \\
u_{t, i}
\end{array}\right]+l_{t, i}^{T}\left[\begin{array}{l}
x_{t, i} \\
u_{t, i}
\end{array}\right]+c_{t, i}\right) \\
& +\frac{1}{2} x_{N_{i}, i}^{T} \hat{P}_{i+1} x_{N_{i}, i}-\hat{\Psi}_{i+1}^{T} x_{N_{i}, i}+\hat{c}_{i+1} \\
\text { s.t. } & x_{0, i}=\hat{x}_{i} \\
& x_{t+1, i}=A_{t, i} x_{t, i}+B_{t, i} u_{t, i}+a_{t, i}, t \in \mathbb{Z}_{0, N_{i}-1},
\end{aligned}
$$

using $p+1$ individual Riccati recursions. Here $Q_{t, i}, l_{t, i}, c_{t, i}$, $A_{t, i}, B_{t, i}$ and $a_{t, i}$ are defined consistently with $\mathrm{x}_{\mathrm{i}}$ and $\mathrm{u}_{\mathrm{i}}$.

Remark 2: The subproblems in (6) do not have any terminal constraints as in the subproblems in [14]. Here the coupling is instead given by the terminal state cost.

\section{B. Eliminate local variables in a subproblem}

It will now be shown that even when $\hat{P}_{i+1}, \hat{\Psi}_{i+1}$ and $\hat{c}_{i+1}$ are not known, it is possible to work on the individual subproblems to eliminate local variables and reduce their sizes. This can be done separately for the $p+1$ subproblems, which opens up for a structure that can be solved in parallel. The core idea with this approach is that the unknowns $\hat{P}_{i+1}$ and $\hat{\Psi}_{i+1}$ will indeed influence the solution of the subproblem, but as soon will be shown the resulting degree of freedom is often very limited compared to the dimension of the full control signal vector $\mathrm{u}_{\mathrm{i}}$. The constant $\hat{c}_{i+1}$ affects the optimal value of the cost function but not the solution.

The following lemma gives an expression for the cost-togo at state $x_{t, i}$ in (6) for some $t$ when the control inputs $u_{\tau, i}$ for $\tau \in \mathbb{Z}_{t, N-1}$ are computed in a certain way.

Lemma 1: Consider a UFTOC problem in the form in (6). Let $P_{\tau, i}, G_{\tau, i}, K_{\tau+1, i}, \Psi_{\tau, i}, k_{\tau+1, i}$ and $\bar{c}_{\tau, i}$ be computed by algorithms 1 and 2 for fixed $\hat{P}_{i+1}, \hat{\Psi}_{i+1}$ and $\hat{c}_{i+1}$, respectively. Furthermore, let $u_{\tau, i}$ be computed as

$$
u_{\tau, i}=k_{\tau+1, i}+K_{\tau+1, i} x_{\tau, i}+\bar{u}_{\tau, i}, \tau \in \mathbb{Z}_{t, N-1},
$$

where $\bar{u}_{\tau, i} \in \mathbb{R}^{n_{u}}$ is an arbitrary vector. Then the cost-to-go at a state $x_{t, i}$ in (6) is given by

$$
\begin{aligned}
\bar{V}\left(x_{t, i}, \overline{\mathrm{u}}_{\mathrm{i}}\right) & \triangleq \frac{1}{2} x_{t, i}^{T} P_{t, i} x_{t, i}-\Psi_{t, i}^{T} x_{t, i}+\bar{c}_{t, i}+ \\
& \frac{1}{2} \sum_{\tau=t}^{N-1} \bar{u}_{\tau, i}^{T} G_{\tau+1, i} \bar{u}_{\tau, i} .
\end{aligned}
$$

Proof: For the proof of Lemma 1, see Appendix A. In the remaining part of this section the subindices $i$ in (6) are omitted for notational brevity, i.e., $\hat{\Psi}_{i+1}$ is written $\hat{\Psi}$ etc.

It will now be shown how local variables in a subproblem can be eliminated by exploiting the structure in the subproblem (6). A preliminary feedback given by the Riccati recursion will be used to simplify the reduction of the subproblems. The use of this preliminary feedback is in principle not necessary, but it will later be seen that some computationally demanding key computations can be performed more efficiently by using it. Let the UFTOC problem (6) be factored and solved for $\hat{P}=0, \hat{\Psi}=0$ and $\hat{c}=0$ using algorithms 1 and 2 . The resulting optimal control law for $\hat{P}=0$ and $\hat{\Psi}=0$ is then $u_{0, t}=k_{0, t+1}+K_{0, t+1} x_{t}$ for $t \in \mathbb{Z}_{0, N-1}$. The subindex " 0 " denotes variables that correspond to this preliminary solution.

It will now be investigated how the control signal $u_{t}$ and the cost function are affected when $\hat{P} \neq 0, \hat{\Psi} \neq 0$ and $\hat{c} \neq 0$. Let the contribution to the control signal $u_{t}$ from the unknown $\hat{P}$ and $\hat{\Psi}$ be denoted $\bar{u}_{t} \in \mathbb{R}^{n_{u}}$. Using the preliminary feedback, which is optimal for $\hat{P}=0$ and $\hat{\Psi}=0$, the control law can be written

$$
u_{t}=k_{0, t+1}+K_{0, t+1} x_{t}+\bar{u}_{t}, t \in \mathbb{Z}_{0, N-1} .
$$

Note that $\bar{u}_{t}$ is an arbitrary $n_{u}$-vector, hence there is no loss of generality in this assumption. From now on, the control law (9) is used in the subproblem, and it will be shown that the degree of freedom in $\bar{u}_{t}$ can be reduced. By defining

$$
\begin{aligned}
& \overline{\mathrm{Q}}_{\overline{\mathrm{u}}} \triangleq\left[\begin{array}{ccc}
G_{0,1} & & \\
& \ddots & \\
& & G_{0, N}
\end{array}\right], \mathrm{A} \triangleq\left[\begin{array}{c}
I \\
\vdots \\
\prod_{t=0}^{N-1}\left(A_{t}+B_{t} K_{0, t+1}\right)
\end{array}\right] \\
& \mathrm{B} \triangleq\left[\begin{array}{cccc}
0 & 0 & \ldots & 0 \\
B_{0} & & & \\
\vdots & & \\
\prod_{t=1}^{N-1}\left(A_{t}+B_{t} K_{0, t+1}\right) B_{0} & & \ldots & B_{N-1}
\end{array}\right]_{(10 \mathrm{~b}}^{(10 \mathrm{a})} \\
& \mathrm{a} \triangleq\left[\begin{array}{c}
0 \\
a_{0}+B_{0} k_{0,1} \\
\vdots \\
\sum_{\tau=0}^{N-1} \prod_{t=\tau+1}^{N-1}\left(A_{t}+B_{t} K_{0, t+1}\right)\left(a_{\tau}+B_{\tau} k_{0, \tau+1}\right)
\end{array}\right],
\end{aligned}
$$

and using (9) the states $x$ can be expressed

$$
\mathrm{x}=\mathrm{A} x_{0}+\mathrm{B} \overline{\mathrm{u}}+\mathrm{a},
$$

where $\bar{u} \in \mathbb{R}^{N n_{u}}$. The cost function when the control law (9) is used is given by the cost-to-go at $x_{0}$ given by Lemma 1 with the terminal cost $\hat{P}=0, \hat{\Psi}=0$ and $\hat{c}=0$, i.e.,

$$
\bar{V}\left(x_{0}, \overline{\mathrm{u}}\right)=\frac{1}{2} x_{0}^{T} P_{0,0} x_{0}-\Psi_{0,0}^{T} x_{0}+\frac{1}{2} \overline{\mathrm{u}}^{T} \overline{\mathrm{Q}}_{\overline{\mathrm{u}}} \overline{\mathrm{u}}+\bar{c}_{0,0} .
$$

Here $P_{0,0}, \Psi_{0,0}$ and $\bar{c}_{0,0}$ are computed by algorithms 1 and 2 with the choice $\hat{P}=0, \hat{\Psi}=0$ and $\hat{c}=0$.

Let the last block rows in $\mathrm{A}$, a and $\mathrm{B}$ be denoted as $\hat{A}, \hat{a}$ and $\mathrm{S}$. The dynamics equations from $x_{0}$ to $x_{N}$ are then

$$
x_{N}=\hat{A} x_{0}+\mathrm{S} \overline{\mathrm{u}}+\hat{a} .
$$

The terminal cost given by $\hat{P},-\hat{\Psi}$ and $\hat{c}$ in (6) is (possibly) non-zero. Hence, the total cost in (6) is obtained by adding the non-zero terminal cost to the cost function $\bar{V}\left(x_{0}, \overline{\mathrm{u}}\right)$ in (12). The UFTOC problem (6) is then equivalently

$$
\begin{aligned}
\min _{x_{0}, \overline{\mathrm{u}}, x_{N}} & \frac{1}{2} x_{0}^{T} P_{0,0} x_{0}-\Psi_{0,0}^{T} x_{0}+\frac{1}{2} \overline{\mathrm{u}}^{T} \overline{\mathrm{Q}}_{\overline{\mathrm{u}} \overline{\mathrm{u}}+\bar{c}_{0,0}} \\
& +\frac{1}{2} x_{N}^{T} \hat{P} x_{N}-\hat{\Psi}^{T} x_{N}+\hat{c} \\
\text { s.t. } & x_{0}=\hat{x} \\
& x_{N}=\hat{A} x_{0}+\mathrm{S} \overline{\mathrm{u}}+\hat{a},
\end{aligned}
$$


where the dynamics equations in (13) have been used. The problem (14) is a UFTOC problem with prediction horizon 1 and $N n_{u}$ control signals and this problem is obtained similarly as in partial condensing, [19]. The equations that define the factorization of the KKT system of (14) are given by

$$
\begin{aligned}
\bar{F} & =P_{0,0}+\hat{A}^{T} \hat{P} \hat{A}, \\
\overline{\mathrm{G}} & =\overline{\mathrm{Q}}_{\overline{\mathrm{u}}}+\mathrm{S}^{T} \hat{P} \mathrm{~S}, \quad \overline{\mathrm{H}}=\hat{A}^{T} \hat{P} \mathrm{~S}, \\
\overline{\mathrm{G}} \overline{\mathrm{K}} & =-\overline{\mathrm{H}}^{T}, \quad \overline{\mathrm{G}} \overline{\mathrm{k}}=\mathrm{S}^{T}(\hat{\Psi}-\hat{P} \hat{a}) .
\end{aligned}
$$

These can be used to compute the optimal solution of (14). Using (15b), the first equation in (15c) can be written as

$$
\left(\overline{\mathrm{Q}}_{\overline{\mathrm{u}}}+\mathrm{S}^{T} \hat{P} \mathrm{~S}\right) \overline{\mathrm{K}}=-\mathrm{S}^{T} \hat{P} \hat{A} .
$$

It will now be shown that it is possible to reduce the number of equations by exploiting the structure in (16). To do this, let $U_{1} \in \mathbb{R}^{N n_{u} \times n_{1}}$ with $n_{1} \leq n_{x}$ be an orthonormal basis for $\mathcal{R}\left(\mathrm{S}^{T}\right)$, i.e., the range space of $\mathrm{S}^{T}$, and let $U_{2}$ be an orthonormal basis of $\mathcal{R}\left(\mathrm{S}^{T}\right)^{\perp}$, both given by the singular value decomposition of $\mathrm{S}^{T}$, i.e.,

$$
\mathrm{S}^{T}=\left[\begin{array}{ll}
U_{1} & U_{2}
\end{array}\right]\left[\begin{array}{cc}
\Sigma_{S} & 0 \\
0 & 0
\end{array}\right]\left[\begin{array}{l}
V_{1}^{T} \\
V_{2}^{T}
\end{array}\right]=U_{1} \Sigma_{S} V_{1}^{T} .
$$

Then $U=\left[\begin{array}{ll}U_{1} & U_{2}\end{array}\right]$ is an orthonormal basis for $\mathbb{R}^{N n_{u}}$, and by using the identity $U U^{T}=I$ and the definitions

$$
\Sigma \triangleq \Sigma_{S} V_{1}^{T} \hat{P} V_{1} \Sigma_{S}, \quad \Gamma \triangleq-\Sigma_{S} V_{1}^{T} \hat{P} \hat{A}
$$

eq. (16) can equivalently be written

$$
\left(U U^{T} \overline{\mathrm{Q}}_{\overline{\mathrm{u}}}+U_{1} \Sigma U_{1}^{T}\right) \overline{\mathrm{K}}=U_{1} \Gamma .
$$

Multiplying (19) by $U_{1}^{T}$ and $U_{2}^{T}$, respectively, from left gives

$$
\begin{aligned}
& \left(U_{1}^{T} \overline{\mathrm{Q}}_{\overline{\mathrm{u}}}+\Sigma U_{1}^{T}\right) \overline{\mathrm{K}}=\Gamma, \\
& U_{2}^{T} \overline{\mathrm{Q}}_{\overline{\mathrm{u}}} \overline{\mathrm{K}}=0 \Longleftrightarrow \overline{\mathrm{K}}=\overline{\mathrm{Q}}_{\overline{\mathrm{u}}}^{-1} U_{1} \hat{K} .
\end{aligned}
$$

In (20b) the matrix $\hat{K} \in \mathbb{R}^{n_{1} \times n_{x}}$ is introduced to parametrize the nullspace of $U_{2}^{T}$ as $U_{1} \hat{K}$. Hence, the freedom in $\overline{\mathrm{K}}$ is described as $\overline{\mathrm{K}}=\overline{\mathrm{Q}}_{\overline{\mathrm{u}}}^{-1} U_{1} \hat{K}$ and inserting this in (20a) gives

$$
\left(U_{1}^{T} \overline{\mathrm{Q}}_{\overline{\mathrm{u}}}+\Sigma U_{1}^{T}\right) \overline{\mathrm{Q}}_{\overline{\mathrm{u}}}^{-1} U_{1} \hat{K}=\left(I+\Sigma U_{1}^{T} \overline{\mathrm{Q}}_{\overline{\mathrm{u}}}^{-1} U_{1}\right) \hat{K}=\Gamma .
$$

This parametrization and reduction of equations can be performed independently of the unknown $\hat{P}$ and $\hat{\Psi}$. Multiplying (21) with $U_{1}^{T} \overline{\mathrm{Q}}_{\overline{\mathrm{u}}}^{-1} U_{1} \in \mathbb{S}_{++}^{n_{1}}$ from the left gives

$$
\left(U_{1}^{T} \overline{\mathrm{Q}}_{\overline{\mathrm{u}}}^{-1} U_{1}+U_{1}^{T} \overline{\mathrm{Q}}_{\overline{\mathrm{u}}}^{-1} U_{1} \Sigma U_{1}^{T} \overline{\mathrm{Q}}_{\overline{\mathrm{u}}}^{-1} U_{1}\right) \hat{K}=U_{1}^{T} \overline{\mathrm{Q}}_{\overline{\mathrm{u}}}^{-1} U_{1} \Gamma .
$$

Using (17) and the definition of $\Sigma$ and $\Gamma$, (22) can be written

$$
\left(U_{1}^{T} \overline{\mathrm{Q}}_{\overline{\mathrm{u}}}^{-1} U_{1}+U_{1}^{T} \overline{\mathrm{Q}}_{\overline{\mathrm{u}}}^{-1} \mathrm{~S}^{T} \hat{P} \mathrm{~S} \overline{\mathrm{Q}}_{\overline{\mathrm{u}}}^{-1} U_{1}\right) \hat{K}=-U_{1}^{T} \overline{\mathrm{Q}}_{\overline{\mathrm{u}}}^{-1} \mathrm{~S}^{T} \hat{P} \hat{A} \text {. }
$$

Now, by introducing the variables

$$
\begin{gathered}
\hat{Q}_{u} \triangleq U_{1}^{T} \overline{\mathrm{Q}}_{\overline{\mathrm{u}}}^{-1} U_{1}, \quad \hat{B} \triangleq \mathrm{S} \overline{\mathrm{Q}}_{\overline{\mathrm{u}}}^{-1} U_{1}, \\
\hat{G} \triangleq \hat{Q}_{u}+\hat{B}^{T} \hat{P} \hat{B}, \quad \hat{H} \triangleq \hat{A}^{T} \hat{P} \hat{B},
\end{gathered}
$$

eq. (23) can be written as

$$
\hat{G} \hat{K}=-\hat{H}^{T} .
$$

Remark 3: The preliminary feedback in (9) results in a block-diagonal $\bar{Q}_{\bar{u}}$ with blocks given by $G_{0, t+1}$ for $t \in$ $\mathbb{Z}_{0, N-1}$. Hence, $\hat{Q}_{u}$ and $\hat{B}$ can be computed efficiently using block-wise computations where the factorizations of $G_{0, t+1}$ from computing $K_{0, t+1}$ can be re-used.

By using analogous calculations the structure can be exploited also in the second equation in (15c) to reduce it to

$$
\hat{G} \hat{k}=\hat{B}^{T}(\hat{\Psi}-\hat{P} \hat{a}),
$$

with $\hat{k} \in \mathbb{R}^{n_{1}}$. Hence, by also defining the variables

$$
\hat{F} \triangleq \bar{F}, \quad \hat{Q}_{x} \triangleq P_{0,0}, \quad \hat{l}_{x} \triangleq-\Psi_{0,0},
$$

the eqs. (15) can now be written as

$$
\begin{aligned}
\hat{F} & =\hat{Q}_{x}+\hat{A}^{T} \hat{P} \hat{A}, \\
\hat{G} & =\hat{Q}_{u}+\hat{B}^{T} \hat{P} \hat{B}, \quad \hat{H}=\hat{A}^{T} \hat{P} \hat{B}, \\
\hat{G} \hat{K} & =-\hat{H}^{T}, \quad \hat{G} \hat{k}=\hat{B}^{T}(\hat{\Psi}-\hat{P} \hat{a}),
\end{aligned}
$$

which can be identified as the factorization of the KKT system of a UFTOC problem in the form (14) but with control signal dimension $n_{\hat{u}}=n_{1} \leq n_{x}$. Hence (29) define the optimal solution to a smaller UFTOC problem. This reduction was performed by eliminating local variables in the problem (6). This important result is summarized in Theorem 1 (where the subindices $i$ in (6) are again used).

Theorem 1: A UFTOC problem given in the form (6) with unknown $\hat{P}_{i+1}, \hat{\Psi}_{i+1}$ and $\hat{c}_{i+1}$ can be reduced to a UFTOC problem in the form

$$
\begin{aligned}
\min _{x_{0, i}, x_{N_{i}, i}, \hat{u}_{i}} & \frac{1}{2} x_{0, i}^{T} \hat{Q}_{x, i} x_{0, i}+\frac{1}{2} \hat{u}_{i}^{T} \hat{Q}_{u, i} \hat{u}_{i}+\hat{l}_{x, i}^{T} x_{0, i}+\hat{c}_{i} \\
& +\frac{1}{2} x_{N_{i}, i}^{T} \hat{P}_{i+1} x_{N_{i}, i}-\hat{\Psi}_{i+1}^{T} x_{N_{i}, i}+\hat{c}_{i+1} \\
\text { s.t. } \quad & x_{0, i}=\hat{x}_{i} \\
& x_{N_{i}, i}=\hat{A}_{i} x_{0, i}+\hat{B}_{i} \hat{u}_{i}+\hat{a}_{i},
\end{aligned}
$$

where $\hat{x}_{i}, x_{0, i}, x_{N_{i}, i} \in \mathbb{R}^{n_{x}}$ and $\hat{u}_{i} \in \mathbb{R}^{n_{\hat{u}}}$, with $n_{\hat{u}} \leq n_{x}$. $\hat{A}_{i}$ and $\hat{a}_{i}$ are the last block rows in $\mathrm{A}_{\mathrm{i}}$ and $\mathrm{a}_{\mathrm{i}}$ given in (10a) and (10c), respectively, and $\hat{Q}_{x, i}, \hat{Q}_{u, i}, \hat{l}_{x, i}$ and $\hat{B}_{i}$ are given by (24) and (28), and $\hat{c}_{i} \triangleq \bar{c}_{0,0}$ where $\bar{c}_{0,0}$ is defined in (12).

Proof: Theorem 1 follows directly from the derivations that are presented in the text in Section IV-B.

To avoid computing the orthonormal basis $U_{1}$ and $U_{2}$ in practice a transformation $\hat{K}=T \hat{L}$, where $T \in \mathbb{R}^{n_{1} \times n_{x}}$ has full rank and $U_{1} T=\mathrm{S}^{T}$, can be used. By using this choice of $\hat{K}$ in (23) and then multiplying from the left with $T^{T}$, the matrices $\hat{Q}_{u}, \hat{B}$ and (26) can instead be written

$$
\hat{Q}_{u}=\hat{B} \triangleq \mathrm{S} \bar{Q}_{\bar{u}}^{-1} \mathrm{~S}^{T} \text { and } \hat{G} \hat{L}=-\hat{H}^{T},
$$

where $\hat{G}$ and $\hat{H}$ are defined as in (25) but with the new $\hat{Q}_{u}$ and $\hat{B}$. The UFTOC problem corresponding to (29) then obtains an (possibly) increased control signal dimension $n_{\hat{u}}=n_{x} \geq n_{1}$ compared to when $\hat{Q}_{u}$ and $\hat{B}$ are defined 
as in (24), but with the advantage that $\hat{Q}_{u}$ and $\hat{B}$ can be easily computed. Analogous calculations can be made for $\hat{k}$.

Remark 4: If $\mathrm{S}^{T}$ is rank deficient then $U_{1} \in \mathbb{R}^{N n_{u} \times n_{1}}$ has $n_{1}<n_{x}$ columns. Hence $\hat{G}$ is singular and $\hat{L}$ non-unique in (31). How to cope with this is described in, e.g., [5], [17].

For the last subproblem $i=p$ the variables $\hat{P}_{p+1}=$ $Q_{x, N_{p}, p}, \hat{\Psi}_{p+1}=-l_{x, N_{p}, p}$ and $\hat{c}_{p+1}=c_{N_{p}, p}$ in (6) are known. Hence, the last subproblem can be factored exactly and all variables but the initial state can be eliminated.

The formal validity of the reduction of each subproblem $i \in \mathbb{Z}_{0, p-1}$ is given by Theorem 1 , while the computational procedure is summarized in Algorithm 4, which is basically a Riccati factorization and backward recursion as in algorithms 1 and 2. Here $\hat{Q}_{u, i}$ and $\hat{B}_{i}$ are computed as in (31).

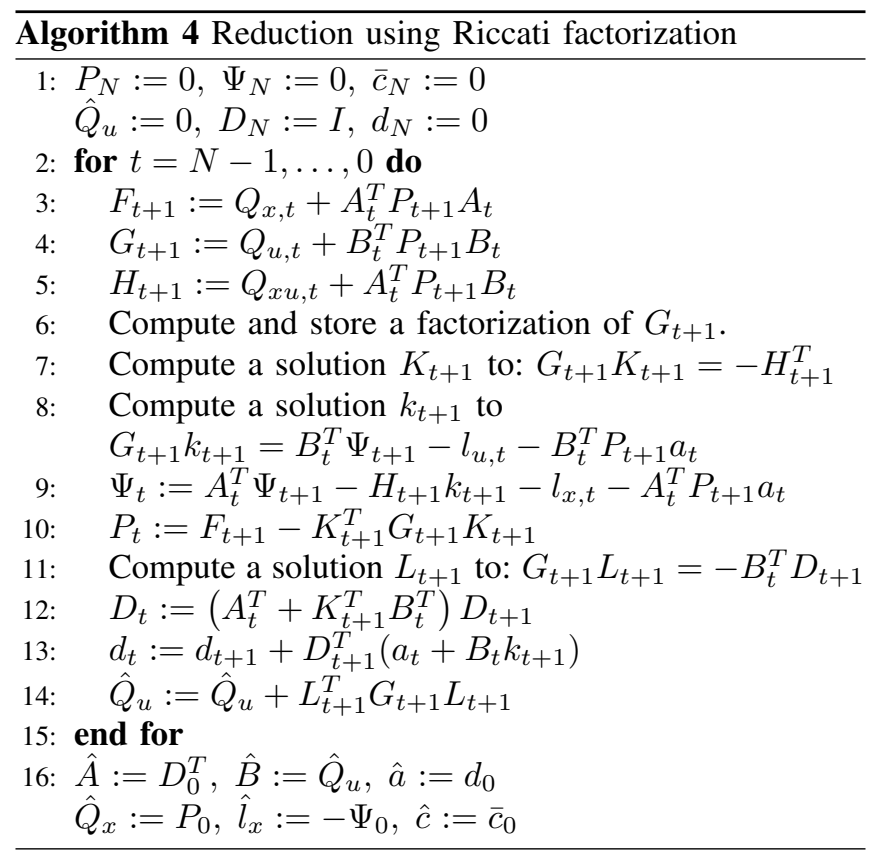

\section{Constructing the master problem}

According to Theorem 1 and Section IV-B all subproblems $i \in \mathbb{Z}_{0, p-1}$ can be reduced to depend only on $\hat{x}_{i}, x_{N_{i}, i}$ and $\hat{u}_{i}$, and subproblem $i=p$ depends only on $\hat{x}_{p}$. The variable $\hat{u}_{i}$ represents the unknown part of the control signals $u_{t, i}$ that are due to the initially unknown $\hat{P}_{i+1}$ and $\hat{\Psi}_{i+1}$ and can be interpreted as a new control signal for batch $i$. Using the definition of the subproblems and the property $x_{N_{i}, i}=x_{0, i+1}=\hat{x}_{i+1}$ that were introduced in Section IVA, the reduced subproblems $i \in \mathbb{Z}_{0, p}$ can be combined into a master problem which is equivalent to the problem in (2). By using the notation from Section IV-B the master problem can be written

$$
\begin{array}{ll}
\min _{\hat{\mathrm{x}}, \hat{\mathrm{u}}} & \sum_{i=0}^{p-1}\left(\frac{1}{2}\left[\begin{array}{l}
\hat{x}_{i} \\
\hat{u}_{i}
\end{array}\right]^{T} \hat{Q}_{i}\left[\begin{array}{c}
\hat{x}_{i} \\
\hat{u}_{i}
\end{array}\right]+\hat{l}_{x, i}^{T} \hat{x}_{i}+\hat{c}_{i}\right) \\
& +\frac{1}{2} \hat{x}_{p}^{T} \hat{Q}_{x, p} \hat{x}_{p}+\hat{l}_{x, p}^{T} \hat{x}_{p}+\hat{c}_{p} \\
\text { s.t. } & \hat{x}_{0}=\bar{x}_{0} \\
& \hat{x}_{i+1}=\hat{A}_{i} \hat{x}_{i}+\hat{B}_{i} \hat{u}_{i}+\hat{a}_{i}, i \in \mathbb{Z}_{0, p-1} .
\end{array}
$$

This is a UFTOC problem in the same form as (2) but with shorter prediction horizon $p<N$, block-diagonal $\hat{Q}_{i}$ and control signals $\hat{u}_{i}$ in each sample $i$. The dynamics equations $\hat{x}_{i+1}=\hat{A}_{i} \hat{x}_{i}+\hat{B}_{i} \hat{u}_{i}+\hat{a}_{i}$ are due to the relation $x_{N_{i}, i}=x_{0, i+1}$. Hence, a UFTOC problem $\mathcal{P}(N)$ can be reduced using Riccati recursions in each subproblem to a UFTOC problem $\mathcal{P}(p)$ in the same form but with shorter prediction horizon and possibly lower control signal dimension.

\section{Computing the Riccati Recursion in PARAllel}

The reduction of the individual subproblems according to Section IV-B can be performed in parallel. To reach consensus between all subproblems and solve the original problem (2), the master problem (32) can be solved to obtain $\hat{P}_{i+1}, \hat{\Psi}_{i+1}, \hat{c}_{i+1}$ and the optimal $\hat{x}_{i}$ for $i \in \mathbb{Z}_{0, p}$. When these variables are known, the independent subproblems are solved in parallel using algorithms 1-3 with the initial $x_{0, i}=\hat{x}_{i}$, $\hat{P}_{i+1}, \hat{\Psi}_{i+1}$ and $\hat{c}_{i+1}$ for $i \in \mathbb{Z}_{0, p}$.

To compute $\hat{P}_{i+1}, \hat{\Psi}_{i+1}, \hat{c}_{i+1}$ and $\hat{x}_{i}$ the master problem (32) can be solved serially using the Riccati recursion. However, (32) can instead itself be reduced in parallel by repeatedly using the theory presented in Section IV until a UFTOC problem with a prediction horizon of pre-determined length is obtained. This top problem is then solved, and the solution is propagated down until the subproblems of the original problem (2) are solved. This procedure is shown in Fig. 1 where $\mathcal{P}_{i}^{k}\left(N_{i}^{k}\right)$ denotes subproblem $i$ in the form (6) at level $k$ in the tree. The overall procedure is similar to what was done in [14], but the conceptual difference here is the way it is performed using Riccati recursions, which allow for a complete exploitation of problem structure. Since the subproblems at each level can be reduced and solved in parallel and the information flow is between parent and children in the tree, the Riccati recursion can be computed in parallel using the theory proposed in this paper.

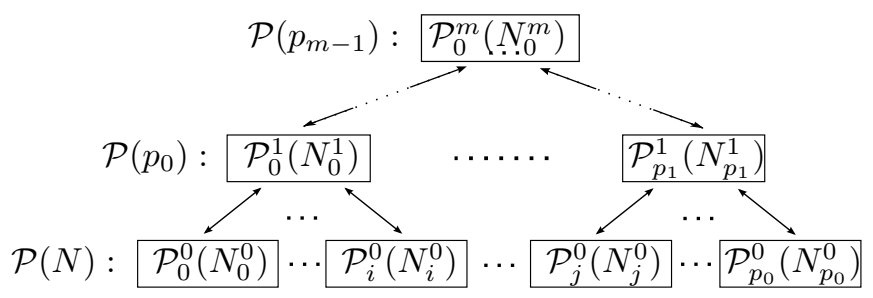

Fig. 1. The original UFTOC problem $\mathcal{P}(N)$ can be reduced repeatedly using Riccati recursions. When the solution to the top problem is computed, it can be propagated back in the tree until the bottom level is solved.

In Algorithm 5, the UFTOC problem (2) is reduced in parallel in several steps to a UFTOC problem with prediction horizon $p_{m-1}$. Assume, for simplicity, that all subproblems are of equal batch length $N_{s}$ and that $N=N_{s}^{m+1}$ for some integer $m \geq 1$. Then the reduction can be made in $m$ steps, provided that $N_{s}^{m}$ computational units are available. Hence, the reduction algorithm has $\mathcal{O}(\log N)$ complexity growth.

When the reduction is complete, each subproblem $i \in$ $\mathbb{Z}_{0, p_{k-1}}$ at level $k$ is solved in Algorithm 6 using algorithms $1-3$ with the optimal $\hat{x}_{i}^{k+1}, \hat{P}_{i+1}^{k+1}, \hat{\Psi}_{i+1}^{k+1}$ and $\hat{c}_{i+1}^{k+1}$ from the respective parent. The algorithm starts by solving 


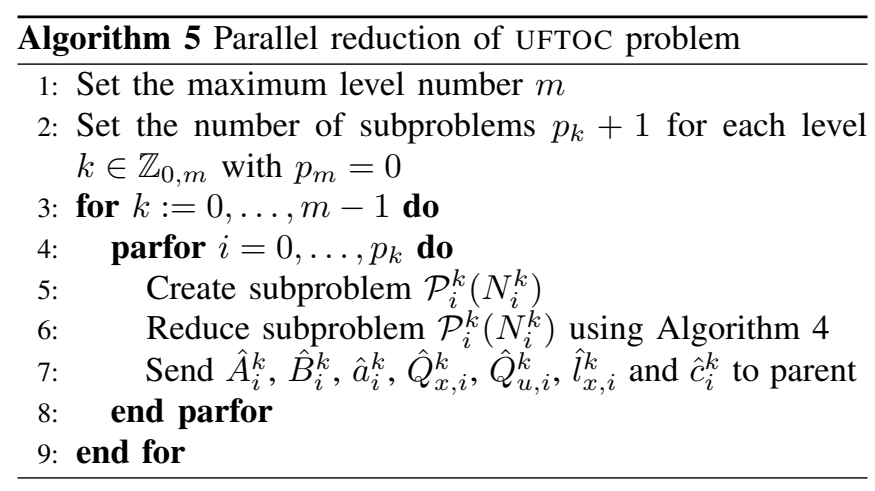

the top problem $\mathcal{P}\left(p_{m-1}\right)$ in Fig. 1, and this solution is propagated to its children. By solving the subproblems at each level and propagating the solution to the level below in the tree, the subproblems $\mathcal{P}_{i}^{0}\left(N_{i}^{0}\right), i \in \mathbb{Z}_{0, p_{0}}$ at the bottom level can finally be solved individually. All subproblems can be solved using only information from their parents, and hence each level in the tree can be solved in parallel.

The optimal primal solution to the original UFTOC problem (2) can be constructed from the solutions to the subproblems using the definition of the local variables. The dual variables can be computed from all $P_{t, i}^{0}$ and $\Psi_{t, i}^{0}$ from the subproblems at the bottom level. Hence there are no complications with non-unique dual variables as in [14] when using the algorithm presented in this paper. The propagation of the solution from the top level to the bottom level can be made in $m+1$ steps provided that $N_{s}^{m}$ processing units are available. Since both algorithms 5 and 6 are solved in $\mathcal{O}(\log N)$ complexity, the Riccati recursion and the solution to (2) can be computed with $\mathcal{O}(\log N)$ complexity growth.

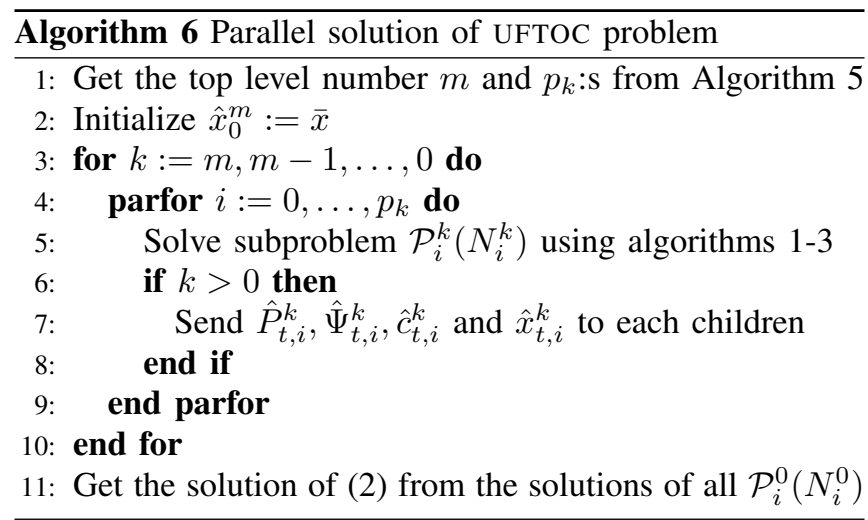

If the algorithms are implemented in double precision arithmetics there are 8 bytes of data per number. Hence, on Line 7 in Algorithm 5 and Line 7 in Algorithm 6 the number of sent bytes is $16 n_{x}^{2}+24 n_{x}+8$ and $4 n_{x}^{2}+20 n_{x}+8$, respectively. In the algorithms in [14] $32 n_{x}^{2}+32 n_{x}+8$ and $24 n_{x}$ bytes of data are sent. The significant decrease in the amount of communication in the algorithm proposed here is due to exploitation of the structure in the subproblems by using Riccati recursions and the relation $\hat{B}_{i}=\hat{Q}_{u, i}$.

Beyond what is presented here, as observed already in [20], standard parallel linear algebra can be used for many computations in the serial Riccati recursion in each subproblem to boost performance even further. This has however not been utilized in this work.

\section{NUMERICAL RESUlTS}

In MATLAB parallel executions of the algorithms are simulated by executing them serially using one computational thread but still using the same information flow as for an actual parallel execution. The total computation time has been estimated by summing over the maximum computation time for each level in the tree, and hence the communication overhead is neglected. The influence of the communication overhead is discussed in the end of this section. The performance of the parallel Riccati algorithm proposed in this work is compared with both the serial Riccati recursion, which is considered a state-of-the-art serial method, and the parallel algorithm presented in [14] (only in MATLAB). In all results presented in this section $N_{s}=2$ has been used.

Remark 5: Different batch lengths can be used for each subproblem in the tree. How to choose these to minimize computation time is not investigated here. However, similarly as in [19], the optimal choice depends on, e.g., the problem and the hardware on which the algorithm is implemented.

In MATLAB the algorithms have been compared when solving problems in the form (2) for systems of dimension $n_{x}=7$ and $n_{u}=5$, and of dimension $n_{x}=20$ and $n_{u}=20$, see Fig. 2. It can be seen that the parallel Riccati algorithm outperforms the serial Riccati for $N \gtrsim 18$ for both problem sizes. The parallel Riccati algorithm is outperforming the parallel algorithm in [14] for both problem sizes, and for $n_{x}=20$ and $n_{u}=20$ the parallel Riccati is approximately three times faster than the algorithm in [14].
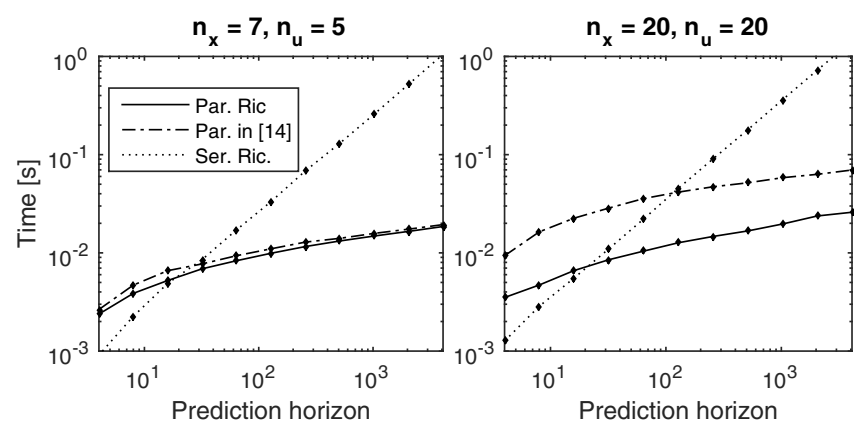

Fig. 2. Computation times using problems of order $n_{x}=20$ and $n_{u}=20$ (left) and $n_{x}=20$ and $n_{u}=20$ (right). The parallel Riccati outperforms the serial Riccati for $N \gtrsim 18$, and it is faster than the algorithm in [14].

In addition to the MATLAB implementation, an ANSI-C implementation has been run on a computational cluster of nodes with 8-core Intel Xeon E5-2660 @ $2.2 \mathrm{GHz}$ CPUs with communication over TCP/IP on Gigabit Ethernet. The computations were performed on resources provided by the Swedish National Infrastructure for Computing (SNIC) at NSC. The implementation is rudimentary and especially the communication setup can be improved. However, the implemented algorithm serves as a proof-of-concept that the algorithm improves performance in terms of computation times for computations on real parallel hardware, taking communication delays into account. The computation times when solving problems in the form (2) for systems of order $n_{x}=20$ and $n_{u}=20$ are seen in Fig. 3, where it is clear that the parallel algorithm solves a problem with 
$N=512$ approximately as fast as the serial algorithm solves the same one for $N=64$, and the break even is at $N \approx 28$. This computational speed-up can be important in problems that require long prediction horizons like, e.g., moving horizon estimation problems, [2], or in high-level planners for autonomous vehicles.

The communication overhead is approximately $20 \%$ for this problem size. It has been observed that communication times are roughly the same regardless of problem size, which indicates that there is a significant communication latency. Reducing these latencies can significantly improve performance of the ANSI-C implemented algorithm.

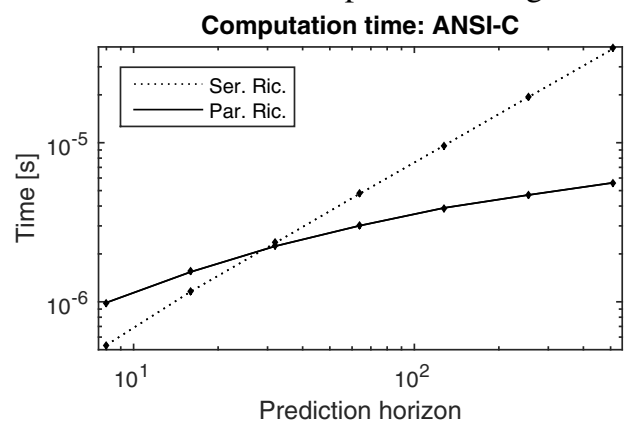

Fig. 3. Computation times for ANSI-C implementation using problems of order $n_{x}=20$ and $n_{u}=20$.

\section{Conclusions}

In this paper it is shown that the Newton step can be solved directly (non-iteratively) in parallel using Riccati recursions that exploit the structure from the MPC problem. A comparison with prior work has been performed in MATLAB, and an ANSI-C implementation of the proposed parallel algorithm has been executed on real parallel hardware. The proposed algorithm obtains logarithmic complexity growth in the prediction horizon length. As future work the possibility to reduce communication latencies by using suitable hardware such as, e.g., Graphics Processing Units (GPUs) or FieldProgrammable Gate Arrays (FPGAs) will be investigated.

\section{A. Proof of Lemma 1 APPENDIX}

The subindices $i$ are omitted for notational brevity in the proof. Assume that (8) holds for an arbitrary $t+1 \in \mathbb{Z}_{1, N-1}$. Then the cost at $t$ is given by

$$
\frac{1}{2}\left[\begin{array}{l}
x_{t} \\
u_{t}
\end{array}\right]^{T} Q_{t}\left[\begin{array}{l}
x_{t} \\
u_{t}
\end{array}\right]+\left[\begin{array}{l}
l_{x, t} \\
l_{u, t}
\end{array}\right]^{T}\left[\begin{array}{l}
x_{t} \\
u_{t}
\end{array}\right]+c_{t}+\bar{V}\left(x_{t+1}, \overline{\mathrm{u}}\right) .
$$

Inserting $x_{t+1}=A_{t} x_{t}+B_{t} u_{t}+a_{t}$ into (33) gives

$$
\begin{aligned}
& \frac{1}{2}\left[\begin{array}{l}
x_{t} \\
u_{t}
\end{array}\right]^{T}\left[\begin{array}{ll}
F_{t+1} & H_{t+1} \\
H_{t+1}^{T} & G_{t+1}
\end{array}\right]\left[\begin{array}{l}
x_{t} \\
u_{t}
\end{array}\right]+\left[\begin{array}{l}
\bar{l}_{x, t} \\
\bar{l}_{u, t}
\end{array}\right]^{T}\left[\begin{array}{l}
x_{t} \\
u_{t}
\end{array}\right]+ \\
& \tilde{c}_{t}+\frac{1}{2} \sum_{t=t+1}^{N-1} \bar{u}_{t}^{T} G_{t+1} \bar{u}_{t},
\end{aligned}
$$

where $F_{t+1}, H_{t+1}$ and $G_{t+1}$ are defined in Algorithm 1 and

$$
\begin{aligned}
& \bar{l}_{x, t} \triangleq l_{x, t}+A_{t}^{T} P_{t+1} a_{t}-A_{t}^{T} \Psi_{t+1}, \\
& \bar{l}_{u, t} \triangleq l_{u, t}+B_{t}^{T} P_{t+1} a_{t}-B_{t}^{T} \Psi_{t+1}, \\
& \tilde{c}_{t} \triangleq \bar{c}_{t+1}+c_{t}+\frac{1}{2} a_{t}^{T} P_{t+1} a_{t}-\Psi_{t+1}^{T} a_{t} .
\end{aligned}
$$

Finally, using the control law $u_{t}=k_{t+1}+K_{t+1} x_{t}+\bar{u}_{t}$ and the definition of $P_{t}, \Psi_{t}, k_{t+1}, K_{t+1}, \bar{l}_{x, t}$ and $\bar{l}_{u, t}$ gives

$$
\begin{aligned}
\bar{V}\left(x_{t}, \overline{\mathrm{u}}\right) & =\frac{1}{2} x_{t}^{T} P_{t} x_{t}-\Psi_{t}^{T} x_{t}+\frac{1}{2} \sum_{t=t}^{N-1} \bar{u}_{t}^{T} G_{t+1} \bar{u}_{t}+\bar{c}_{t}, \\
\bar{c}_{t} & \triangleq \tilde{c}_{t}+\frac{1}{2} k_{t+1}^{T} G_{t+1} k_{t+1}+\bar{l}_{u, t}^{T} k_{t+1} .
\end{aligned}
$$

Eq. (8) holds specifically for $t=N-1$ and hence Lemma 1 follows by induction. This completes the proof.

\section{REFERENCES}

[1] H. Jonson, "A Newton method for solving non-linear optimal control problems with general constraints," Ph.D. dissertation, Linköpings Tekniska Högskola, 1983.

[2] C. Rao, S. Wright, and J. Rawlings, "Application of interior-point methods to model predictive control," Journal of Optimization Theory and Applications, vol. 99, no. 3, pp. 723-757, Dec. 1998.

[3] A. Hansson, "A primal-dual interior-point method for robust optimal control of linear discrete-time systems," IEEE Transactions on Automatic Control, vol. 45, no. 9, pp. 1639-1655, Sep. 2000.

[4] D. Axehill, L. Vandenberghe, and A. Hansson, "Convex relaxations for mixed integer predictive control," Automatica, vol. 46, no. 9, pp. 1540-1545, 2010.

[5] D. Axehill, "Integer quadratic programming for control and communication," Ph.D. dissertation, Linköping University, 2008.

[6] D. Axehill and A. Hansson, "A dual gradient projection quadratic programming algorithm tailored for model predictive control," in Proceedings of the 47th IEEE Conference on Decision and Control, Cancun, Mexico, 2008, pp. 3057-3064.

[7] I. Nielsen, D. Ankelhed, and D. Axehill, "Low-rank modification of Riccati factorizations with applications to model predictive control," in Proceedings of the 52nd IEEE Conference on Decision and Control, Firenze, Italy, Dec. 2013, pp. 3684-3690.

[8] G. Constantinides, "Tutorial paper: Parallel architectures for model predictive control," in Proceedings of the European Control Conference, Budapest, Hungary, 2009, pp. 138-143.

[9] D. Soudbakhsh and A. Annaswamy, "Parallelized model predictive control," in Proceedings of the American Control Conference, Washington, DC, USA, 2013, pp. 1715-1720.

[10] Y. Zhu and C. D. Laird, "A parallel algorithm for structured nonlinear programming," in 5th International Conference on Foundations of Computer-Aided Process Operations, vol. 5, 2008, pp. 345-348.

[11] P. Reuterswärd, Towards Pseudospectral Control and Estimation, ser. ISSN 0280-5316, 2012, licentiate Thesis.

[12] B. O'Donoghue, G. Stathopoulos, and S. Boyd, "A splitting method for optimal control," in IEEE Transactions on Control Systems Technology, vol. 21, no. 6 . IEEE, 2013, pp. 2432-2442.

[13] G. Stathopoulos, T. Keviczky, and Y. Wang, "A hierarchical timesplitting approach for solving finite-time optimal control problems," in Proceedings of the European Control Conference, Zurich, Switzerland, July 2013, pp. 3089-3094.

[14] I. Nielsen and D. Axehill, "An O(log N) parallel algorithm for Newton step computation in model predictive control," in Proceedings of the 19th IFAC World Congress, Cape Town, South Africa, Aug. 2014, pp. $10505-10511$.

[15] — "An O(log N) parallel algorithm for Newton step computation in model predictive control," arXiv preprint arXiv:1401.7882, 2014.

[16] S. Khoshfetrat Pakazad, A. Hansson, and M. Andersen, "Distributed primal-dual interior-point methods for solving loosely coupled problems using message passing," arXiv:1502.06384v2, 2015.

[17] I. Nielsen, On Structure Exploiting Numerical Algorithms for Model Predictive Control, ser. Linköping Studies in Science and Technology. Thesis, 2015, no. 1727.

[18] J. Nocedal and S. Wright, Numerical Optimization. Springer-Verlag, 2006.

[19] D. Axehill, "Controlling the level of sparsity in MPC," Systems \& Control Letters, vol. 76, pp. 1-7, 2015.

[20] D. Axehill and A. Hansson, "Towards parallel implementation of hybrid MPC - a survey and directions for future research," in Distributed Decision Making and Control, ser. Lecture Notes in Control and Information Sciences, R. Johansson and A. Rantzer, Eds. Springer Verlag, 2012, vol. 417, pp. 313-338. 